\title{
Treatment of liver cancer of middle and advanced stages using ultrasound-guided percutaneous ethanol injection combined with radiofrequency ablation: A clinical analysis
}

\author{
XUE SUN ${ }^{1 *}, \mathrm{RU} \mathrm{LI}^{2^{*}}$, BOTAO ZHANG ${ }^{3}$, YUEJIE YANG $^{3}$ and ZHIFEI CUI ${ }^{3}$ \\ ${ }^{1}$ Department of Medical Ultrasonics, The Second Affiliated Hospital of Zhengzhou University; \\ ${ }^{2}$ Department of Medical Ultrasonics, The Third Hospital of Zhengzhou; ${ }^{3}$ Department of Medical Ultrasonics, \\ Zhengzhou Central Hospital Affiliated to Zhengzhou University, Zhengzhou, Henan 450000, P.R. China
}

Received December 18, 2015; Accepted February 2, 2016

DOI: $10.3892 / \mathrm{ol} .2016 .4180$

\begin{abstract}
Liver cancer is a malignancy of the digestive system and has a high morbidity and mortality rate. Local intervention has become a viable option in identifying liver treatment. The aim of the present study was to analyze the clinical effects of treating liver cancer in middle and advanced stages using ultrasound-guided percutaneous ethanol injection (PEI) in tumors combined with radiofrequency ablation (RFA). A total of 100 patients with stage III-IV liver cancers were selected to participate in the study. Patients were divided into groups. In group A, treatment was initiated with PEI and after 1-2 weeks RFA was applied while in group B treatment was initiated with RFA and after 1-2 weeks PEI was applied. Patients in group C received PEI and RFA simultaneously. The clinical effects in the 3 groups were compared after 6-month follow ups. The volume of tumor ablation necrosis in group A was significantly greater than that in the groups $\mathrm{B}$ and $\mathrm{C}$, while the size was significantly smaller compared to groups $\mathrm{B}$ and $\mathrm{C}$ after ablation. For group A, the complete ablation rate was significantly higher than that in groups $\mathrm{B}$ and $\mathrm{C}$, and the differences were statistically significant $(\mathrm{P}<0.05)$. Liver damage indices, including raising levels of glutamic-pyruvic transaminase and total bilirubin, were significantly decreased in group A $(\mathrm{P}<0.05)$. The survival rate in group $\mathrm{A}$ was also significantly higher than in groups $\mathrm{B}$ and $\mathrm{C}(\mathrm{P}<0.05)$. In conclusion, for patients with liver cancer in middle and advanced stages, the treatment method using PEI followed by RFA was more
\end{abstract}

Correspondence to: Dr Zhifei Cui, Department of Medical Ultrasonics, Zhengzhou Central Hospital Affiliated to Zhengzhou University, 195 Tongbai Road, Zhengzhou, Henan 450000, P.R. China

E-mail: cui_zhifei1@163.com

*Contributed equally

Key words: percutaneous ethanol injection, radiofrequency ablation, liver cancer of middle and advanced stages, survival time beneficial in terms of improving the tumor ablation rate, alleviating liver damages and increasing survival rates.

\section{Introduction}

Liver cancer is a malignant tumor of the digestive system with the highest morbidity and mortality rate in China. Early symptoms are often not typical and clinical diagnoses usually occur during middle and advanced stages. Consequently, the surgical resection rate is extremely low at $5-20 \%$ (1). Local tumor intervention treatment has become a viable option for the treatment of liver cancer. In addition, ultrasound-guided percutaneous ethanol injection (PEI) in tumors and percutaneous radiofrequency ablation (RFA) in early stage and small hepatocellular carcinoma have become increasingly applied. These methods were shown to be effective for obtaining a comparable clinical effect compared to surgical resections (2), and also have other advantages such as negligible trauma, extremely low rates of complications and are readily accepted by doctors and patients. However, in cases of tumor ablation without the background of 'pseudocapsule' there is a risk of alcohol diffusion during the injection which may damage the normal liver tissue (3). RFA also has a few advantages including 'thermal subsidence' and 'three-dimensional leakage effect'. Nevertheless, it is not effective in cases of larger tumors and tumors located in particular areas of the body, such as the aorta, diaphragmatic surface, gall bladder and heart (4).

Several studies on the application of PEI in combination with RFA for the treatment of small hepatocellular carcinoma and liver cancer are available (5). However, the application of PEI in combination with RFA to treat liver cancer in middle and advanced stages has yet to be examined.

\section{Patients and methods}

Patients. Between March 2013 and March 2015, 100 patients diagnosed with stage III-IV (Union for International Cancer Control stages) liver cancer were selected. The study conformed to interventional treatment testimony. Approval was obtained from the Ethics Committee of Zhengzhou Central Hospital 
Affiliated to Zhengzhou University and informed consent was provided by patients and their families.

The inclusion criteria for the study were: i) Patients were mentally conscious; ii) all tumors were $<8 \mathrm{~cm}$ in diameter and the total number of tumors was $<6$; and iii) it was the first time patients were treated using PEI and RFA. The exclusion criteria for the study were: i) Patients with hepatic metastasis; ii) pregnant patients and those with serious coagulation mechanism disorders as well as those with medical histories of liver cancer resection and chemoradiotherapy and serious cachexia; iii) patients who had $<6$ months survival expectancy; and iv) patients who were intolerant to surgery and those with interrupted treatments, and missed follow ups.

According to the selective treatment plan, the patients were divided into 3 groups. In group A ( $n=35)$, treatment was initiated with PEI and after 1-2 weeks RFA was applied, while in group $B(n=33)$ treatment was initiated with RFA and after 1-2 weeks PEI was applied. Patients in group $C$ received PEI and RFA simultaneously. The clinical effects in the 3 groups were compared after 6 months.

Group A, comprised 20 male and 15 female patients, with an age range of 56-72 years and an average age of $63.4 \pm 10.5$ years. The mean tumor size was $4.6 \pm 1.3 \mathrm{~cm}$ (range, $3.0-5.5 \mathrm{~cm}$ ) and the total number of tumors was 1-4 with an average of $2.7 \pm 1.3$. There were 16 patients with liver function classification Child-Pugh B and 19 patients with Child-Pugh C, of which 5 cases had tumors in paricular regions and 30 cases had other conditions. In group B, there were 17 male and 16 female patients, with an age range of 55-71 years and an average age of $61.7 \pm 12.3$ years. The mean tumor size was $4.3 \pm 1.4 \mathrm{~cm}$ (range, $2.8-5.6 \mathrm{~cm}$ ) and the total number of tumors was $1-4$ with an average of $2.3 \pm 1.5$. In group B there were 18 patients with liver function classification Child-Pugh B and 15 patients with Child-Pugh C, of which 4 cases had tumors in particular areas and 29 patients had other conditions. Group C, comprised of 19 male and 13 female patients with an age range of 54-75 years and average age of $63.6 \pm 15.2$ years. The mean tumor size was $4.7 \pm 1.2 \mathrm{~cm}$ (range, $3.5-6.0 \mathrm{~cm}$ ) and the total number of tumors was $1-4$ with an average of $2.3 \pm 1.5$. There were 17 patients with liver function classification Child-Pugh B and 15 patients with Child-Pugh $\mathrm{C}$ of which 5 cases of tumors were in particular areas and 27 patients had other conditions. The comparisons of baseline information for the 3 groups showed that the differences were not statistically significant $(\mathrm{P}>0.05)$.

Treatments. The patient was placed in supine position and PEI was performed under general anesthesia, using percutaneous puncture needling guided by ultrasound (Aloka SSD-1100 Color Doppler Ultrasound; Siemens AG, Munich, Germany). Local anesthesia was performed using lidocaine. A $22 \mathrm{G} \mathrm{EV}$ needle (Hakko Co., Ltd., Tokyo, Japan) was entered into the tumor by percutaneous liver biopsy, no blood and gail were evident when the syringe was withdrawn. Using a $10 \mathrm{ml}$ syringe, anhydrous alcohol was uniformly injected into the tumor within 30-60 sec. After the alcohol injection, a high echogenic mass was observed under ultrasound that spread from the pinpoint. Injections were stopped when the high echogenic mass fully covered the tumor and surpassed the edge by $>0.5 \mathrm{~cm}$. The injection volume was calculated using the formula: $V=4 / 3 \pi(r+0.5)^{3}$ where $V$ is total volume, and $r$ is the radius of lesion. After completion of injection, the puncture needle was gradually withdrawn. Drug overflow was avoided as the procedure was observed under ultrasound. After withdrawal of the puncture needle, the puncture point was covered with gauze and pressured was applied on the dressing (Fig. 1).

RFA was performed under general anesthesia once the patient was in supine position, using percutaneous puncture needling guided by ultrasound (Aloka SSD-1100 Color Doppler Ultrasound; Siemens AG). Local anesthesia was performed using lidocaine. The cool-tip RF System (Valleylab, Boulder, CO, USA), uni-polar cold circulation system, mating electrode wire and plate electrode were used during the process. Output frequency was adjusted to $(1 \pm 5 \%) 460 \mathrm{kHz}$, the maximum power was $150 \mathrm{~W}$ and the needle used was a $14 \mathrm{G}$ trocar. A total of 7-12 fine needle electrodes were set on top of the inner needles, which formed a $5.0 \mathrm{~cm}$ spherical heat coagulation after stretching energization. The unit was set to $90 \mathrm{~W}$ for RFA actual burning power and the temperature was set at $100^{\circ} \mathrm{C}$. After the temperature reached $100^{\circ} \mathrm{C}$, it was maintained for $15 \mathrm{~min}$. Following completion of a single RFA, the echo of the ablation area under the observation of ultrasound was higher, the position of the needle electrodes was adjusted according to the tumor size for repeated ablation, in order to cover the whole target tumor in the echo area, and the ablation area surpassed tumor lesions by $0.5-1 \mathrm{~cm}$. Following completion of the treatment, the stretching electrodes were withdrawn, and the needles were withdrawn after burning of closed needles. The adjustments of radiofrequency power started from $60 \mathrm{~W}$ each time, and it were gradually elevated until impedance markedly increased and power was automatically reduced. The scope of vaporization of strong echo covering all boundaries of the tumors was used to detrermine when to terminate the treatment. To prevent hemorrhage and implantation metastasis, needles were heated under high temperature each time prior to use (Fig. 2).

Observation indices. The volume of tumor ablation necrosis, volume after ablation and complete ablation rate, quantity of alcohol and radiofrequency energy used and liver function damage indices, including raising levels of glutamic-pyruvic transaminase and total bilirubin were recorded. Differences of survival rates were compared 6 months after follow-ups. To determine tumor volume the formula used was: $\mathrm{V}\left(\mathrm{cm}^{3}\right)=4 / 3$ x r1 $(\mathrm{cm}) \times \mathrm{r} 2(\mathrm{~cm}) \times \mathrm{r} 3(\mathrm{~cm})$, (r1=longest diameter/2, r2=shortest diameter/2, r3=height/2). The volume of tumor necrosis was calculated by subtracting the volume after ablation from the volume before ablation. A contrast-enhanced ultrasound examination was conducted two weeks after the treatment. Arterial portal and delayed phases of the ablation area with no contrast agent observed was regarded as complete ablation; radiofrequency energy $(\mathrm{J})=$ watt $(\mathrm{W}) \mathrm{x}$ curative time (sec).

Statistical analysis. SPSS 20.0 software (IBM SPSS, Armonk, NY, USA) was used for statistical analyses. Measurement data were presented by mean \pm standard deviation. Analysis of variance was used for comparisons among the groups and enumeration data were presented by $\%$, while the $\chi^{2}$ test was used for comparison among groups. $\mathrm{P}<0.05$ was considered to indicate a statistically significant difference. 
Table I. Comparisons among different ablation parameters.

\begin{tabular}{lccccccc}
\hline Groups & Cases & $\begin{array}{c}\text { Volume before } \\
\text { ablation }\left(\mathrm{cm}^{3}\right)\end{array}$ & $\begin{array}{c}\text { Volume after } \\
\text { ablation }\end{array}$ & $\begin{array}{c}\text { Volume of necrosis } \\
\text { ablation }\end{array}$ & $\begin{array}{c}\text { Complete ablation } \\
\text { rate (\%) }\end{array}$ & $\begin{array}{c}\text { Usage volume } \\
\text { of alcohol, ml }\end{array}$ & $\begin{array}{c}\text { Radiofrequency } \\
\text { energy }\left(\mathrm{x} 10^{3} \mathrm{~J}\right)\end{array}$ \\
\hline $\mathrm{A}$ & 35 & $15.5 \pm 3.3$ & $1.5 \pm 0.8$ & $13.8 \pm 4.2$ & $30(85.7)$ & $8.5 \pm 1.3$ & $456.7 \pm 33.4$ \\
$\mathrm{~B}$ & 33 & $14.7 \pm 3.4$ & $6.6 \pm 1.2$ & $10.6 \pm 3.7$ & $20(60.6)$ & $8.2 \pm 1.5$ & $432.6 \pm 45.2$ \\
$\mathrm{C}$ & 32 & $15.3 \pm 3.5$ & $7.2 \pm 1.5$ & $10.5 \pm 3.8$ & $20(62.5)$ & $8.3 \pm 1.6$ & $423.4 \pm 43.3$ \\
$\mathrm{~F}\left(\chi^{2}\right)$ & & 0.632 & 6.926 & 6.524 & 6.360 & 0.423 & 0.938 \\
$\mathrm{P}$-value & 0.425 & 0.032 & 0.038 & 0.042 & 0.725 & 0.546 \\
\hline
\end{tabular}

Group A, PEI was administered first and then RFA; group B, RFA was administered first and then PEI; group C, PEI and RFA were administered simultaneously. PEI, percutaneous ethanol injection; RFA, radiofrequency ablation.

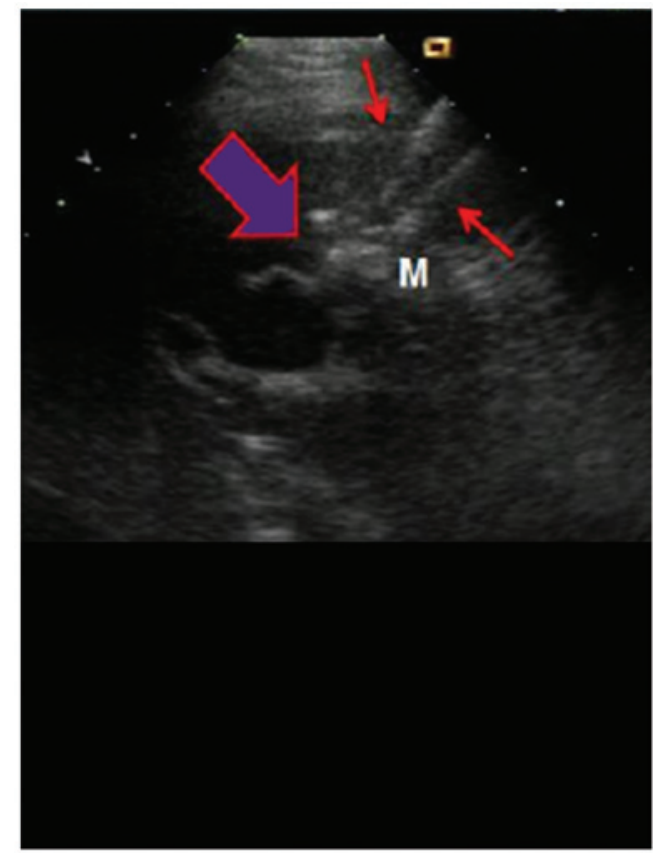

Figure 1. The process of percutaneous ethanol injection.

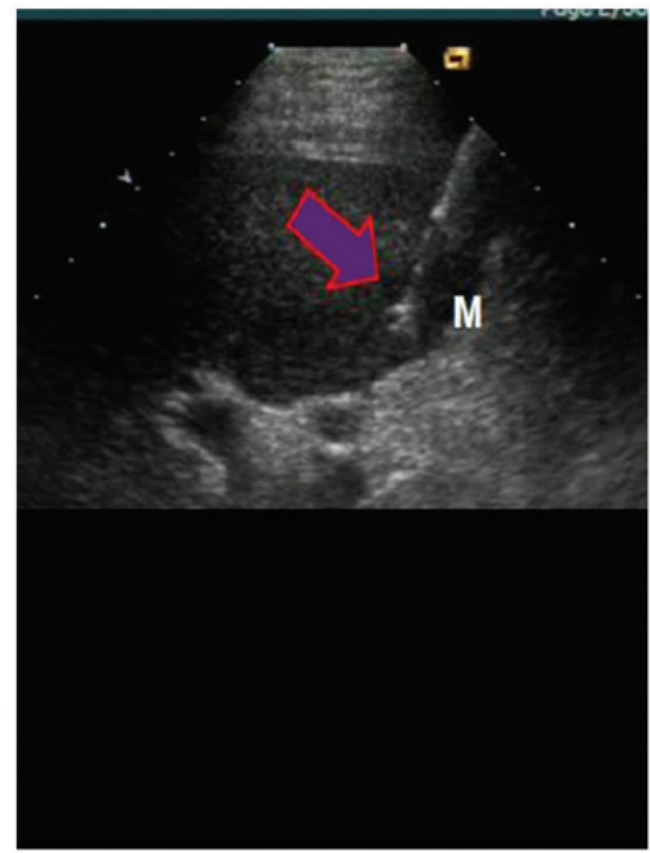

Figure 2. The process of radiofrequency ablation.

\section{Results}

Comparisons of ablation parameters. The volume of tumor ablation necrosis in group A was significantly larger than that in groups $\mathrm{B}$ and $\mathrm{C}$, while the volume was significantly smaller than that in groups $\mathrm{B}$ and $\mathrm{C}$ after ablation. The complete ablation rate in group $\mathrm{A}$ was significantly higher than that in groups B and C, and the differences were statistically significant $(\mathrm{P}<0.05)$. The comparisons of quantity of alcohol used and radiofrequency energy in the three groups showed that the differences were not statistically significant $(\mathrm{P}>0.05)$ (Table I).

Comparisons of liver function damage indices. The comparisons of glutamic-pyruvic transaminase and total bilirubin levels prior to the treatment in the 3 groups showed that the differences were not statistically significant $(\mathrm{P}>0.05)$. By contrast, after the treatment, the raising levels of glutamic-pyruvic transaminase and total bilirubin in group A were significantly lower than that in groups $\mathrm{B}$ and $\mathrm{C}$, and the differences were statistically significant $(\mathrm{P}<0.05)$ (Table II).

Comparisons of survival rates. Eight patients succumbed in group A, of whom 2 had tumor recurrence in situ, 1 patient succumbed to tumor rupture hemorrhage, 3 succumbed to tumor emboli blocking the hepatic artery or portal vein hemorrhage, 3 patients succumbed to liver failure, and 1 patient had extrahepatic tumor metastasis. Mortalities occured 2 weeks to 6 months after the treatment (average, 4.2 months) and the survival rate was $77.1 \%$. In group B, 17 patients succumbed of whom 5 patients had tumor recurrence in situ, 3 patients succumbed to tumor rupture hemorrhage, 7 patients to tumor emboli blocking the hepatic artery or portal vein hemorrhage, 5 due to liver failure, and 2 patients succumbed to extrahepatic tumor metastasis. In group B, mortalities occured 1 week to 5.5 months after treatment (average, 3.5 months), and the survival rate was $48.5 \%$. In group C, 15 mortalities occured of whom 6 patients succumbed to tumor recurrence in situ, 
Table II. Comparisons among liver function damage indices.

\begin{tabular}{|c|c|c|c|c|}
\hline Groups & $\begin{array}{l}\text { Glutamic-pyruvic transaminase } \\
\text { level before treatments, U/1 }\end{array}$ & Raising levels ${ }^{\mathrm{a}}$ & $\begin{array}{l}\text { Total bilirubin before } \\
\text { treatments, } \mu \mathrm{mol} / 1\end{array}$ & Raising levels ${ }^{\mathrm{a}}$ \\
\hline A & $78.5 \pm 13.6$ & $13.6 \pm 4.2$ & $27.9 \pm 5.3$ & $6.3 \pm 1.2$ \\
\hline B & $75.6 \pm 14.2$ & $18.2 \pm 4.7$ & $25.5 \pm 4.2$ & $10.2 \pm 2.5$ \\
\hline $\mathrm{C}$ & $76.7 \pm 13.5$ & $17.5 \pm 4.3$ & $23.4 \pm 4.6$ & $8.9 \pm 2.4$ \\
\hline F-value & 0.632 & 6.325 & 0.759 & 6.856 \\
\hline P-value & 0.421 & 0.039 & 0.532 & 0.027 \\
\hline
\end{tabular}

${ }^{\mathrm{a} B e f o r e ~ t r e a t m e n t ~-~ a f t e r ~ t r e a t m e n t . ~}$

4 to tumor rupture hemorrhage, 6 to tumor emboli blocking the hepatic artery or portal vein hemorrhage, 3 to liver failure, and 2 patients succumbed to extrahepatic tumor metastasis. Patients in group $\mathrm{C}$ succumbed to the disease 1.5 weeks to 5.6 months following treatment (average, 3.7 months), and the survival rate was $53.1 \%$. The survival rate in group A increased significantly, and the differences were statistically significant $\left(\chi^{2}=6.739, \mathrm{P}=0.034\right)$.

\section{Discussion}

Ultrasound guidance technology has the advantages of real-time monitoring, accurate guidance, negligible trauma, safety and effectiveness, easy operation and repeatability (6). These advantages have led this technology to be considered as one of the three major types of treatment for liver cancer together with surgery and regional vascular intervention (7). Ultrasound guidance technology aims to effectively treat tumors or cytoreduction, reducing symptoms, improving life quality and extending survival time.

Ultrasound-guided local interstitial chemical ablation consists of injecting substances into tumors to induce necrosis. Frequently used injections include anhydrous alcohol, acetic acid, hot saline water or hot distilled water, radionuclide, biological agents and cod liver oil acid sodium anhydrous alcohol solution (8). Sugiura et al (9) clinically applied PEI in treating liver cancer for the first time, and now PEI is the most widely used method for chemical ablation. However, due to the disadvantages of non-uniform diffusion and uncontrollability of injected alcohol observed in numerous treatments of larger tumors, PEI is mainly applied in the treatment of small hepatocellular carcinoma. However, Livraghi et al (10) reported that PEI could be applied in $\geq 5 \mathrm{~cm}$ liver tumors. They showed that for the 1,066 patients participating in their study, the 3-, 5- and 7-year survival rates were 72.3, 43.2 and 27.0\%, respectively. The ultrasound-guided local interstitial thermal ablation method consists of conducting energy into the tumor to inactivate tumor cells in situ. Thermal ablation includes radiofrequency, microwave, laser, refrigeration and focused ultrasound. Rossi et al (11) were the first group who reported treating small hepatocellular carcinoma using ultrasound-guided RFA, with comparable results compared to PEI. RFA can also be used as a supplementary method for the treatment of large liver tumors. RFA is also suitable for those patients that cannot tolerate surgery or those unwilling to undergo surgery. The results obtained from prior studies showed that in cancer tissues, HSP-70 expression level, AFP expression level, clinical staging, liver function classifications and the inactivated degree of tumors in liver cancer tissues, were are all closely associated with RFA treatment effects in large liver tumors (12). RFA was certified by the Food and Drug Association in early 1996 and currently is cosidered the most widely applied ablation method (13). There are some limitations associated with RFA such as high costs, limited popularity and technological difficulties.

The application of PEI in combination with RFA for the treatment of large liver cancer and liver cancer of particular areas has become a hot topic of research. This method is designed to reduce the tumor size of through dehydration and solidification using anhydrous alcohol, and thus increase the success rate of RFA ablation and reduce the frequency of ablation (14). Multi-polar RFA treatment devices (RITA Medical System; Rita Medical, Mountain View, CA, USA; Radio-Therapeutics, Sunnyvale, CA, USA) have 3-5 electrodes that are similar to 'eagle claw' or 'umbrella'. The cold circulation in radiofrequency electrode is designed to prevent the ebullition and the formation of cavities of tissues around the pinpoint of the electrodes because of the heat (15). There are no absolute conclusions regarding whether PRI and RFA should be carried out seperately or simultaneously, and there are few studies on the quantitative comparison of the volume of alcohol usage and radiofrequency power. Through large numbers of clinical practices, it was identified that although the basis of liver cancer in middle and advanced stages is poor, survival time cannot be improved via conservative treatments (16). The clinical effects of simple PRI and RFA treatments are not substantial, however, the present study concluded that adimistering PEI treatment 1-2 weeks prior to RFA treatment can significantly improve the patients' condition by intensification of the volume of tumor ablation necrosis and reduce tumor volume following ablation. This method can also improve complete the ablation rate, reduce the raising levels of glutamic-pyruvic transaminase and total bilirubin and improve the survival rate. The volume of alcohol usage and radiofrequency power was not increased in this method. Possible reasons for this include: i) The stress damage of anhydrous alcohol to organic bodies is higher than that of RFA, and PEI was used for the first time (17); ii) usually there is the existence of 'pseudocapsule' in primary liver cancer, and PEI being used for the first time, which influences the dispersion 
effects of anhydrous alcohol (18); iii) the working mechanism of RFA is to use ion in tissues to create vibrations of the same frequency around electrodes, which turns it into heat energy to produce coagulative necrosis. The pretreatment of anhydrous alcohol can make the frequency of tumor tissues more concentrated, which shortens the duration of RFA (19); and iv) the simultaneous implementations of PEI and RFA treatments cannot produce a superimposed effect, which in turn creates the phenomenon of offset (20).

In conclusion, for patients with liver cancer in middle and advanced stages, the treatment method using PEI followed by RFA is more beneficial in terms of improving tumor ablation rate, alleviating liver damage and increasing survival rates.

\section{References}

1. Hou W and Zhu X: Extra vascular interventional treatment of liver cancer, present and future. Drug Discov Ther 9: 335-341, 2015.

2. Xu LT, Zhou ZH, Lin JH, Chen Z, Wang K, Wang P, Zhu XY, Shen YH, Meng ZQ and Liu LM: Clinical study of transarterial chemoembolization combined with 3-dimensional conformal radiotherapy for hepatocellular carcinoma. Eur J Surg Oncol 37: 245-251, 2011.

3. Kurokohchi K, Watanabe S, Masaki T, Hosomi N, Miyauchi Y, Himoto T, Kimura Y, Nakai S, Deguchi A, Yoneyama H, et al: Comparison between combination therapy of percutaneous ethanol injection and radiofrequency ablation and radiofrequency ablation alone for patients with hepatocellular carcinoma. World J Gastroenterol 11: 1426-1432, 2005

4. Mulier S, Ni Y, Jamart J, Ruers T, Marchal G and Michel L: Local recurrence after hepatic radiofrequency coagulation: Multivariate meta-analysis and review of contributing factors. Ann Surg 242: 158-171, 2005.

5. Jarnagin WR: Management of small hepatocellular carcinoma: A review of transplantation, resection, and ablation. Ann Surg Oncol 17: 1226-1233, 2010

6. Kim JW, Shin SS, Heo SH, Hong JH, Lim HS, Seon HJ, Hur YH, Park CH, Jeong YY and Kang HK: Ultrasound-guided percutaneous radiofrequency ablation of liver tumors: how we do it safely and completely. Korean J Radiol 16: 1226-1239, 2015.

7. Zhang TT, Luo HC, Cui X, Zhang W, Zhang LY, Chen XP and Li KY: Ultrasound-guided percutaneous microwave ablation treatment of initial recurrent hepatocellular carcinoma after hepatic resection: long-term outcomes. Ultrasound Med Biol 41: 2391-2399, 2015

8. Clark TW: Chemical ablation of liver cancer. Tech Vasc Interv Radiol 10: 58-63, 2007.
9. Sugiura Y, Iwasaka T and Tarumi N: Percutaneous ethanol injection therapy: A new treatment for hepatocellular carcinoma. Radiaology 90: 53-57, 1983.

10. Livraghi T, Giorgio A, Marin G, Salmi A, de Sio I, Bolondi L, Pompili M,BrunelloF, Lazzaroni S and Torzilli G: Hepatocellular carcinoma and cirrhosis in 746 patients: Long-term results of percutaneous ethanol injection. Radiology 197: 101-108, 1995.

11. Rossi S, Di Stasi M, Buscarini E, Cavanna L, Quaretti P, Squassante E, Garbagnati F and Buscarini L: Percutaneous radiofrequency interstitial thermal ablation in the treatment of small hepatocellular carcinoma. Cancer J Sci Am 1: 73-81, 1995.

12. Kudo M: Radiofrequency ablation for hepatocellular carcinoma: Updated review in 2010. Oncology 78 (Suppl 1): 113-124, 2010.

13. Wahl DR, Stenmark MH, Tao Y, Pollom EL, Caoili EM, Lawrence TS, Schipper MJ and Feng M: Outcomes After Stereotactic Body Radiotherapy or Radiofrequency Ablation for Hepatocellular Carcinoma. J Clin Oncol: Nov 30, 2015 (Epub ahead of print)

14. Kai L, Jia L, Zhi-Gang W and Lei Y: Ultrasonic guided percutaneous ethanol injection with or without combined radiofrequency ablation for hepatocellular carcinomas. Indian J Cancer 52 Suppl: E102-E104, 2015.

15. Rehman J, Landman J, Lee D, Venkatesh R, Bostwick DG, Sundaram C and Clayman RV: Needle-based ablation of renal parenchyma using microwave, cryoablation, impedance- and temperature-based monopolar and bipolar radiofrequency, and liquid and gel chemoablation: laboratory studies and review of the literature. J Endourol 18: 83-104, 2004.

16. Ogasawara S, Chiba T, Ooka Y, Suzuki E, Kanogawa N, Saito T, Motoyama T, Tawada A, Kanai F and Yokosuka O: Post-progression survival in patients with advanced hepatocellular carcinoma resistant to sorafenib. Invest New Drugs 14: $1-3,2016$

17. Shi F, Tan Z, An H, Wang X, Xu Y and Wang S: Hepatocellular carcinoma $\leq 4 \mathrm{~cm}$ treated with radiofrequency ablation with or without percutaneous ethanol injection. Ann Hepatol 15: 61-70, 2015-2016.

18. Fu Y, Zhao X, Yun Q, Zhu X, Zhu Y, Li Q, Hu K, Wang J and Qiao Z: Transarterial chemoembolization (TACE) plus percutaneous ethanol injection (PEI) for the treatment of unresectable hepatocellular carcinoma: A meta-analysis of randomized controlled trials. Int J Clin Exp Med 8: 10388-10400, 2015.

19. Yang B, Zan RY, Wang SY, Li XL, Wei ML, Guo WH, You X, Li J and Liao ZY: Radiofrequency ablation versus percutaneous ethanol injection for hepatocellular carcinoma: a meta-analysis of randomized controlled trials. World J Surg Oncol 13: 96, 2015.

20. Huang H, Liang P, Yu XL, Cheng ZG, Han ZY, Yu J and Liu FY: Safety assessment and therapeutic efficacy of percutaneous microwave ablation therapy combined with percutaneous ethanol injection for hepatocellular carcinoma adjacent to the gallbladder. Int J Hyperthermia 31: 40-47, 2015. 